\title{
Supplemental Nutrition Assistance Program (SNAP) Recommendations to Address Healthy Food Access in the United States
}

\author{
Victor Romano ${ }^{1 *}$, Jennifer Chambers ${ }^{1}$, Miracle Etim Andy ${ }^{1}$, Matthew Arnold ${ }^{1}$, and Stephanie Quintanilla ${ }^{2}$ \\ ${ }^{1}$ Department of Sport \& Health Sciences, Catawba College, North Carolina, USA
}

${ }^{2}$ Department of Biology, Catawba College, North Carolina, USA

*Corresponding author: Victor Romano, Department of Sport \& Health Sciences,

Catawba College, Salisbury, North Carolina, USA.

Received Date: July 29, 2020

Published Date: October 30, 2020

\begin{abstract}
The Supplemental Nutrition Assistance Program (SNAP) is a federally funded program to provide food assistance to struggling Americans. The SNAP program, currently under review, is proposing a nearly $30 \%$ decrease, thus eliminating benefits for at least 4 million people and reducing benefits for many others. The current SNAP program only provides $\$ 1.40$ per meal per person, leading to families rely on foods that are less expensive, and often provide less nutritional value, to stretch out their monthly allotment. The new SNAP proposal would drastically cut 260,000 store options around the country, in favor of a new government-driven approach of providing boxed food for SNAP households. SNAP participants are currently at a significant disadvantage in terms of dietary quality relative to non-participants in fruit and vegetable consumption. This policy change would further increase this issue. Food insecurity, even marginal food security, is associated with chronic and costly health problems and behaviors among adults. SNAP participants may be at a higher risk of having a nutritional intake pattern of low consumption of all the five major food groups, while also consuming larger than recommended amounts of fats, sugars, and sodium. Not only are SNAP participants consuming less across all main food groups, their dietary quality is less than non-SNAP participants including whole grains, leafy green vegetables, and healthy meats, due to costs. With the SNAP program currently under review, we recommend the following policy changes,
\end{abstract}

- Increase participant availability through innovative options to increase consumption of fresh fruits, vegetables, and whole grains,

- Increase benefit disbursal to promote more frequent purchases with the goal to increase purchases of fresh fruit, vegetables and healthy meats, and

- Develop and implement nutrition education strategies to educate participants about spending their benefits on more nutritious, cost-effective meals.

Keyword: SNAP; Healthy Food; Food Policy Recommendation

\section{SNAP Eligibility}

The Supplemental Nutrition Assistance Program (SNAP), is a federally funded program to provide food assistance to those that struggle to afford it. This program provides timely, targeted, and temporary benefits to people in need. SNAP has helped millions of low-income Americans have the necessary nutritional support and is one of the largest programs working to fight hunger. As changes happen to the population, the SNAP program changes to meet needs. Although SNAP was created to help those in assistance, only certain people are eligible [1].

To be eligible to receive SNAP benefits, the income for a household must be a certain amount. Because SNAP is targeted for the citizens most at-risk, predominantly serving households with children, elderly or disable members, it is limited to people with gross income up to 130 percent which is what the government 
considers the poverty line. This means that families with up to four members can make no more than 2,633 dollars a month if they want benefits from the SNAP program. Able-bodied adults without dependents can only receive three months of benefits during any three-year period if they're not working a minimum of 20 hours per week or participating in a training program. The law claims that it allows states to confer "categorical eligibility" on those that have been eligible for similar means-tested programs like the Temporary Assistance for Needy Families (TANF) [2].

There are many concerns for the SNAP program. Studies have found that the current issues for this program one example is Block granting SNAP, which typically provides a fixed amount of federal funding for a state and is then that state (not the Federal Government) takes responsibility for determining how the program is operated. This gives States flexibility to determine the program's policies to their specific needs and circumstances, there are tradeoffs on a positive note. If funding is fixed, the program may no longer respond to increased needs, for example during an economic downturn. Also, in the absence of national eligibility standards, it's possible that two people with identical circumstances may receive different benefits because they are in a different State [3].

Another example of an issue would be the limited type of food participants can buy. Limiting the sources of food a participant can purchase not only takes out the consumers' choice but it can reduce SNAP participants. Although this restriction does make consumers eat healthier, it may not be as effective as wanted if the number of SNAP participants lower and can also be ineffective if bought with their own money. Store eligibility is another one, to become authorized to use SNAP, a food retailer must follow the eligibility requirements which is constantly being modified. This can make stores change what is being sold in their stores by having healthier sources for the participants in the SNAP program. Although this can seem as a good thing, it can also be an issue as this can lower the number of stores that can be authorized and also can decrease the number of future participating stores [4].

In July 2019, the U.S Department of Agriculture (USDA) Food and Nutrition Service (FNS) created a loophole that allows people that would have not been eligible, able to participate. Some states have expanded the use of categorical eligibility, this was done so that more households who receive SNAP may participate in these other programs. For example, they could receive an information brochure, a "benefit" which is often given without determining income or assets. This is why SNAP financial eligibility standards are not determined the same way for households in states that have adopted the "expanded categorical eligibility" or "broad based categorical eligibility". As a result, households that would not be eligible under regular program rules would be qualified to participate in the program [5]
In summary, the SNAP program is a federal funded program to assist low-income families. It is a program to fight hunger in America and although it has many benefits, eligibility takes a certain amount of income and not just anybody can be authorized. The SNAP program has concerns like block granting SNAP, limited food sources, and store eligibility. There are suggested improvements to make the program better. One proposed solution was to revise SNAPs categorical eligibility, this would let more people participate and provide more help for low-income homes. The SNAP program is a good way to fight hunger, but eligibility seems to be an issue for participants.

\section{Health Equity Gap of People on SNAP}

Health equity means that all groups of people are afforded the same chance and opportunity to be healthy. Some sources report that SNAP helps to minimize health inequity by providing assistance with the cost of health and nutritious foods that participants may have not been able to get otherwise. On average, SNAP is able to provide participants with $\$ 1.40$ per meal per person (Carlson). While this may seem like a small amount, it is said to have benefited its participants with not only the resources to purchase food, but food security and better health. As an effect, participants and their families have to spend less on health care. One study found that the cost of health care decreased by about $25 \%$ or $\$ 1,4005$.

On the other hand, some research shows that SNAP is nothelping to improve the health equity of low-income families. There are still barriers that keep families that rely on SNAP from reaching their full health potential. The issues occur are concerns of obesity and other chronic diseases, financial stability, lack of access to health foods due to lack of time, transportation, or shopping centers, and a lack of knowledge and support with healthy eating habits [6].

In terms of food stability, SNAP only provides a little support. Research found that participants had to search for assistance outside of the help SNAP provided [7]. Having to use other resources, such as borrowing money adds extra stress to the participant. For some families, paying for bills is more important than worrying about the kind of food they will eat. This being said families rely on foods that are less expensive and often provide less nutrition. Food lacking in proper nutrition and the over consumption of food, especially unhealthy foods, can result in obesity. Without fixed regulations on the federal or state level, there is a positive correlation between the amount of years spent on SNAP and obesity [8].

\section{SNAP Participants Lack Nutrient-Dense Food}

Statistically significant disadvantage in terms of diet quality relative to comparable nonparticipants, in the areas of fruit, vegetables, and whole grain products. An intake pattern in which low consumption of all the five major food groups has been observed, and a pattern of consuming large amounts of fats 
and sugars was recorded. It can be assumed that the association between higher weights of participants and program participation gives cause to examine caloric density versus nutrient density of availability of SNAP food [9]. Female food stamp users tend to weigh an average of 11 more pounds than non-food stamp users10. Generally, individuals in low-income households tend to consume diets that promote chronic conditions such as obesity, morbidity, and premature mortality. These individuals' diets have a pattern of low consumption of fruits and vegetables and high consumption of processed foods [10], specifically a decrease of dark green/orange vegetables [11].

\section{Sodium Levels in SNAP Approved Foods}

Processed foods are high in sodium. The top ten high sodium foods consumed by Americans according to the CDC are bread rolls, pizza, sandwiches, cold cuts/cured meats, soups, burritos, tacos, savory snacks, chicken, cheese, eggs, omelets. Excess sodium can increase a person's risk of high blood pressure, which can lead to heart disease and stroke. On average Americans consume over $3400 \mathrm{mg}$ of sodium (equivalent to $1 \frac{1 / 2}{2}$ tsp of salt) daily. According to Guidelines of Adequate Intakes (AI), the AI of sodium is $1500 \mathrm{mg}$ a dad [12]. Whereas the Chronic Disease Risk Reduction (CDDR) suggests an intake of no more than $2300 \mathrm{mg}$ a day [13].

There was a study completed in 2018 that showed and compared dietary trends of SNAP participants and non-SNAP participants. The research showed a slightly lower sodium intake for SNAP participants averaging at 3,100 $\mathrm{mg} / \mathrm{d}$ compared to income eligible nonparticipants who averaged an intake of $3,239 \mathrm{mg} / \mathrm{d}$ and high-income individuals who averaged an intake of 3,330 mg/d. Although SNAP participants are consuming less sodium than the other groups, it is still over double the recommended intake, which is a cause for concern. Processed meat servings consumed were an average 5\% higher for SNAP participants [14]. This study does not indicated participant's purchasing habits, especially those that purchase mostly processed foods, as many participants do this to same time and money, and their respective sodium intake.

\section{SNAP Meat Options}

Health is a challenging concept to define as there is not finite answer that works across the board because all humans react to stimuli differently. The easiest factor to manipulate in terms of health that reaps the greatest benefit is diet. What we consume leads to how our bodies are able to carry out with our daily activities. Animals are similar in this aspect, as what they consume leads to the quality of their products. This stresses the importance of understanding how the nutrition of animals can affect their byproducts and impact individual's nutrition. The, composition of the different meat products can lead to different reactions in our bodies. Beef is often comprised of mainly fat and the easiest way to manipulate this fatty acid composition is through dietary fat, energy level, and forage concentrates [15]. By highlighting better quality meat by altering their diets, support programs like SNAP can incentivize families to purchase these goods and farmers to raise healthier livestock whose products can improve long term health. However, altered diets can lead to different tastes of meat, reduced shelf life, and metallic taint. The link between FA and heart disease. A Higher intake of Saturated Fatty Acids (FA) has been identified as high-risk factor for heart disease [16].

A diet consisting of high intakes of omega-3 fatty acids is often associated with good heart health. This assumption is correct as the intake of these healthy fats facilitate benefits such as, fetal development, cardiovascular function, Alzheimer's disease, and overall healthy aging. These fatty acids are best consumed through fish and fish-oil supplements. Consuming a healthy amount of these omega-3 fatty acids can aid in long term health as it carries an array of benefits to facilitate a healthy aging process. Omega-3 fatty acids also contain eicosapentaenoic acid (EPA) and docosahexaenoic acid (DHA) which carry the benefits found in pre-natal development and healthy aging [17].

Incentivizing the purchase of fish for the SNAP program can allow those in the program to have the necessary nutrients their bodies need to properly develop [18]. EPA and DHA which is found in large amount in fish, have been found to reduce major coronary artery events and be as effective as aspirin due to the facilitation of blood flow [19]. This stresses the importance of consuming a healthy amount of products that contain these compounds to ensure our bodies have the necessary nutrients to live a healthy life. Ultimately, the SNAP program should incentivize the purchase of products containing these fatty acids.

Wild game is often more difficult to access than farmed meat due to scarcity, but both provide different nutritional benefits primarily due to the manipulation of diets. The manipulation of animal's diets lead to different compositions of meat [20]. A fattier and less active lifestyle will increase bulk on an animal and yields a product with higher fat composition than an active and wild animal. Grass fed beef is found to yield similar meat composition in the wild and farmed conditions. This is most likely due to the similar diets in farmed conditions as well as wild. Wild animals tend to consume higher quality food that contain better nutrients as most diets of the farmed meat are synthetically made such as grains. Farmed animals tend to consume fatty grains which add bulk to help meet the demands of the product [21]. Wild animals do not consume hormones and chemical additives found in the grains. Wild game is leaner and contains larger protein amounts than Farmed meat due to the diet's differences. Wild meat also contains lower amounts of saturated fats but have higher amounts of unsaturated fats which are linked to better brain and heart health [22]. 
Organic and conventional meat have different compositions that can aid in long term health. Organic beef has been found to have " $17 \%$ less cholesterol, $32 \%$ less fat, $16 \%$ less fatty acids, $24 \%$ less monounsaturated fatty acids, $170 \%$ more $\alpha$-linolenic acid, $24 \%$ more $\alpha$-tocopherol, $53 \%$ more $\beta$-carotene, 34\% more coenzyme Q10 and 72\% more taurine than conventional beef." Organic pork reported higher amounts of E coli when compared to conventional pork. "Organic pork also showed lower amounts of antimicrobial resistance in E coli." This antimicrobial aids in reducing the development and spread of this food borne bacteria [23].

Current SNAP recommendations limit what meat sources can be purchased. Fish type and freshness is limited to frozen farm raised. Meat is non-organic and lower quality than that of what is not SNAP approved. Wild game is not covered either as it is considered a luxury purchase, with a loophole of famer markets that do not list the name of meat on the receipt, just that cost of the meat. This is a questionable practice that can get the vendor it trouble depending on the strictness of the oversight organization in charge within their location.

\section{Conclusion}

In December 2019, the United States announced a plan to update the work requirements for SNAP eligibility that would eliminate benefits for an estimated 700,000 people in the United States. The current rule allows states to provide waivers to those who do not meet the minimum work requirement of 20 hours per week for three months in a 36-month period [24]. The new rules would eliminate the power of states to provide waivers for work requirements for able bodied adults ages 18-49 who do not have children and are not disabled. While SNAP may have nutritional limitations and stringent requirements, removing this waiver will effectively eliminate the main source of food for SNAP recipients in low-income areas with high unemployment.

With the SNAP program currently under review and with no chance of removing the work requirement, we recommend the following policy changes to address the issues about the lack of quality of food that is brought up in this paper:

1) Increase participant availability through innovative options to increase consumption of fresh fruits, vegetables, and whole grains.

2) Increase benefit disbursal to promote more frequent purchases with the goal to increase purchases of fresh fruit, vegetables and healthy meats.

3) Develop and implement nutrition education strategies to educate participants about spending their benefits on more nutritious, cost-effective meals.

\section{Acknowledgement}

None.

\section{Conflict of Interest}

Author declare no conflict of interest.

\section{References}

1. B Thorn, C Tadler, N Huret (2018) SNAP is linked with improved nutritional outcomes and lower health care costs. Washington, DC: Center on Budget and Policy Priorities 17: 1-9.

2. Fleischhacker, Sheila, Alyssa Moran, Sara N Bleich (2019) Legislative and executive branch developments affecting the United States Department of Agriculture Supplemental Nutrition Assistance Program. Journal of Food Law \& Policy 15: 131

3. Oliveira, Victor (2018) Design issues in USDA's supplemental nutrition assistance program: Looking ahead by looking back.

4. Wendy Keenan, Clarissa E Sanchez, Erin Kellogg, Sarah M Tracey (2019) Achieving behavioral health equity for children, families, and communities: proceedings of a workshop. National Academies Press.

5. Carlson, Steven, and Brynne Keith Jennings (2018) SNAP is linked with improved nutritional outcomes and lower health care costs. Washington, DC: Center on Budget and Policy Priorities p. 1-19.

6. Azétsop, Jacquineau, Tisha R Joy (2013) Access to nutritious food, socioeconomic individualism and public health ethics in the USA: a common good approach. Philos Ethics and Humanit Med 8: 16.

7. Schenck Fontaine, Anika, Anna Gassman Pines, Zoelene Hill (2017) Use of informal safety nets during the supplemental nutrition assistance program benefit cycle: How poor families cope with within-month economic instability. Social Service Review 91(3): 456-487.

8. Schmeiser, Maximilian D (2012) The impact of long-term participation in the supplemental nutrition assistance program on child obesity. Health e $\backslash$ Economics 21(4): 386-404

9. Michele Ver Ploeg, Lisa Mancino, Biing Hwan Lin, Chia Yih Wang (2007) The vanishing weight gap: trends in obesity among adult food stamp participants (US) (1976-2002). Econ Hum Biol 5(1): 20-36.

10. Seth A Berkowitz, Hilary K Seligman, Joseph Rigdon, James B Meigs, Sanjay Basu (2017) Supplemental Nutrition Assistance Program (SNAP) participation and health care expenditures among low-income adults. JAMA Intern Med 177 (11): 1642-1649.

11. Gleason, Philip, Anu Rangarajan, Christine M Olson Dietary intake and dietary attitudes among food stamp participants and other low-income individuals. Mathematica Policy Research, Incorporated, 2000.

12. Maillot, Matthieu, Adam Drewnowski (2012) A conflict between nutritionally adequate diets and meeting the 2010 dietary guidelines for sodium. Am J Prev Med 42(2): 174-179.

13. Drewnowski Adam, Colin D Rehm, Matthieu Maillot, Alfonso Mendoza, Pablo Monsivais (2015) The feasibility of meeting the WHO guidelines for sodium and potassium: a cross-national comparison study. BMJ Open 5(3): e006625.

14. Zhang Fang Fang, Junxiu Liu, Colin D Rehm, Parke Wilde, Jerold R Mande, et al. (2018) Trends and disparities in diet quality among US adults by Supplemental Nutrition Assistance Program participation status. JAMA Network Open 1(2): e180237-e180237.

15. Costa Ana SH, Marta P Silva, Cristina P M Alfaia, Virgínia M R Pires, Carlos M G A Fontes, et al. (2013) Genetic background and diet impact beef fatty acid composition and stearoyl-CoA desaturase mRNA expression. Lipids 48(4): 369-381.

16. Vargas Bello Pérez, Rafael E Larraín (2017) Impacts of fat from ruminants' meat on cardiovascular health and possible strategies to alter its lipid composition. J Sci Food Agric 97(7): 1969-1978.

17. Swanson Danielle, Robert Block, Shaker A Mousa (2012) Omega-3 fatty acids EPA and DHA: health benefits throughout life. Adv Nutr 3(1): 1-7.

18. (2018) Basics Policy The Supplemental Nutrition Assistance Program (SNAP). Retrieved Sept 9: 2018 
19. Watson, Ronald Ross, ed. Omega-3 fatty acids in brain and neurological health. Elsevier, 2014.

20. Robbins, Charles (2012) Wildlife feeding and nutrition. Elsevier.

21. Provenza Frederick D, Scott L Kronberg, Pablo Gregorini (2019) Is grassfed meat and dairy better for human and environmental health? Front Nutr 6: 26 .

22. Neethling J, LC Hoffman, M Muller (2016) Factors influencing the flavour of game meat: A review. Meat Sci 113: 139-153.
23. Średnicka Tober Dominika, Graham C Burdge , Smaragda Sotiraki, Alexandros Stefanakis, Halil Yolcu, et al. (2016) Composition differences between organic and conventional meat: a systematic literature review and meta-analysis. Br J Nutr 115(6): 994-1011.

24. Neuert, Harrison (2019) Work Requirements Don't Work. A behavioral science perspective. 